\title{
Erratum to: Quantitative Convergence Towards a Self-Similar Profile in an Age-Structured Renewal Equation for Subdiffusion
}

\author{
Hugues Berry ${ }^{1}$. Thomas Lepoutre ${ }^{1,2}$. \\ Álvaro Mateos González ${ }^{1,3}$
}

Published online: 20 October 2016

(C) Springer Science+Business Media Dordrecht 2016

In this article the name of author Álvaro Mateos González was captured incorrectly. Mateos was captured as the second of his given names while in fact it is the first of his two family names.

The online version of the original article can be found under doi:10.1007/s10440-016-0048-3.

$凶$ Á. Mateos González

alvaro.mateos_gonzalez@ens-lyon.fr

H. Berry

hugues.berry@inria.fr

T. Lepoutre

thomas.lepoutre@inria.fr

1 Inria, 56 Blvd Niels Bohr, 69603 Villeurbanne, France

2 Institut Camille Jordan, CNRS UMR 5208, Université de Lyon, Université Claude Bernard Lyon 1, 43 blvd. du 11 novembre 1918, 69622 Villeurbanne cedex, France

3 École Normale Supérieure de Lyon, Unité de Mathématiques Pures et Appliquées, CNRS UMR 5669, Université de Lyon, 15 parvis René Descartes, 69007 Lyon, France 